\title{
Door Bell Notification System for Deaf People using Arduino
}

\author{
C Devi Supraja, V Bindusree
}

\begin{abstract}
This present research paper proposes every problem human race has ever faced, technology has given its answer. Technology has now evolved in each and every aspect of human world, from electric cars to smart washing machine and whatnot. Technology has improved life of differently blind people in many ways too. Our motivation for this project is to help deaf people respond non-speech crucial sounds efficiently so that they can live a normal life. In this project a user module is designed which will notify the user whenever the doorbell is pressed. The notification will be send through a wireless module which covers a good amount of range. One module will be hooked at the doorbell while other will be connected to the user module (a wearable device) with some LED/vibrating motor for the indication purpose. The LCD screen will display the text for notification purpose. These modules are controlled by Arduino control Unit.
\end{abstract}

Keywords-Arduino, Wireless module, LCD screen, vibrating motor, LED, smart washing machine, electric cars.

\section{INTRODUCTION}

Technology has always provided a platform for people with disabilities. From helping a visual impaired read a book to help a mute speak and get included in a conversation. As we are advancing into the technology we are trying to make machines smarter and smarter so that human lives can be improved by a greater extent. The power of innovation is such that now a person can check their fitness level on their mobile application; they can check the quality of sleep just by clicking the application on the mobile phone. These features that the science provides us are so user friendly that people of all age group can utilize its benefit.

We have reached that level in the field of technology that now it's possible to do the impossible. These technologies along with serving its purpose also help to boost the confidence of people who thinks they are helpless and techniques in order to resolve there hearing issues. Thanks to assistive technology they can do or at least try to do things which were once difficult. Technique like Speech therapy helps kids/ adults train their voice so that they can strengthen their voice. Many institutions follow this therapy process for their patients. While on the other hand gadgets like smart gloves translates the American Sign Language into speech so that they can communicate around with other people.

Revised Manuscript Received on October 22, 2019

C Devi Supraja, Dept. of ECE, Institute of Aeronautical Engineering, Hyderabad, India

V Bindusree, Dept. of ECE, Institute of Aeronautical Engineering, Hyderabad, India inferior to others. Not everyone can afford surgical improve upon their pitch, volume which helps them to

The Gesture recognition is done with the help of sensor glove which consists of few accelerometers, a microcontroller and Bluetooth chip which are fixed on the fingers, based on the analysis of American Sign Language signs. The concept of decoding gestures is done by considering the axis orientation with respect to gravity and their corresponding voltage levels are recorded [1].

How harsh it would be to live without one of the senses? This question not only terrifies us to the depth but it also helps us to understand how important they are to the human body. Each and every element of our body is so important that we could never imagine a life without them. During our visit to the deaf and dumb school located in Nadiad, Gujarat made us realize about one question that was, how bad is it to live without hearing anything? Taking this as a challenge and our urge to help those people we thought of developing a smart device help deaf people to fight their problem. The problem targeted was to help deaf people respond to doorbells whenever pressed.

\section{RELATED WORK}

In our day to day life, we come across many non- speech sounds which give us important information about surroundings. Matthews et al. [2] has shown that how important it is to identify various non-speech sounds for deaf people. They conducted interviews with hearing impaired patients and normal people. Some examples of such sounds are friends or co- workers talking in a room, music or instructions given in public place, cry of children and critical information like fire alarm, cell phone ring or doorbelling. Sound visualization for deaf people [3], in this paper various techniques has been analyzed which are used to create sound awareness amongst deaf people. Techniques which were analyzed are vibration sensing, Flashing lights, Hearing dogs, Cochlear implants. The chart for the same is show below

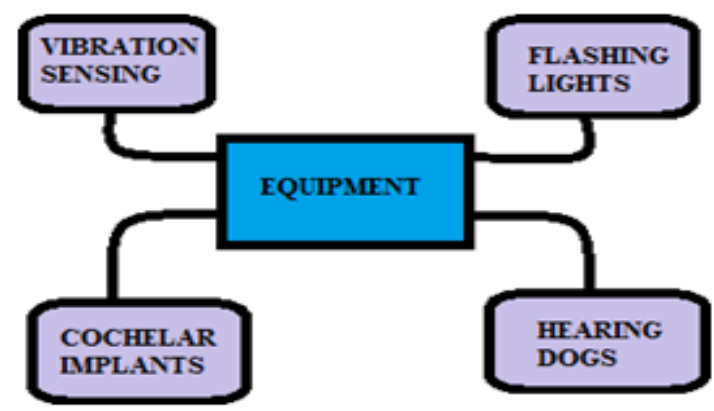

Fig.1. various sound awareness techniques. [3] 
Vibration sensing creates awareness of sound to create vibration, for instance if someone is walking nearby the user, the equipment will sense it and then the vibration will be generated which will notify the user. The major drawback with such systems is that it depends on the infrastructure of the house; wooden flooring will be better sensed then concrete tiles. Flashing lights helps user to create awareness of telephonic, doorbell sound. This approach requires the equipment to be hooked up at user's side. Hearing dogs won't have much impact in order to create the awareness of the surrounding. This method first of all requires ongoing maintenance and secondly the dog has to been trained for each and every possible sound. Still one cannot guarantee about this method on how effective will it be. Last method is cochlear implants; this for no doubt increase the sensitivity of the ears but not everyone in the world can afford it. Apart from this, implantation can cause other health issues like nerve damage which causes paralyses, loss of hearing that the patient previously had, ringing in ears and many more.

After analyzing all the methods we finalized to develop a notifying system based on flashing LED and vibrating motor based approach. Murniwati BT Anwar designed Wireless notification system for the hearing impaired [4]. This project used PIC as a microcontroller along with an encoder and decoder to control several operations like baby cry notification, doorbell notification and etc. Author has implemented many important notifications, making the whole system very big. Wristband unit is also heavy miking difficult for user to wear for the full day. It has limitations in working in dense home environment. Using Arduino over PIC can be quite advantageous. Arduino covers all the complexity of microcontrollers. Arduino IDE provides a great place to write and code for embedded systems. One can select different boards, different port, can include any library for the IDE itself. To program an Arduino one simply needs a USB cable, while to program PIC a separate PIC programmer is required to upload the code. Simplicity in Arduino's architecture and in its user friendly environment gives Arduino an upper hand than PIC. In this proposed paper, the objective is achieved using Arduino as a microcontroller. Also the device developed is compact in size and thus it is wearable.

\section{SYSTEM DESCRIPTIONS}

Smart doorbell notifying system aims to simplify the lives of deaf people. Keeping our target in mind which was to develop low cost, light in weight device which could be a wearable one, we developed device helps the disabled do basic things which occurs almost daily. Imagine a situation where a person with hearing impairment is home alone, and someone knocks the door or rings a bell, since the person cannot hear the knock or doorbell, the door won't be unlocked as a result the visitor may go away. The situation would have been different if the person inside would have some portable/wearable device on which the notification would have been popped up whenever the doorbell would have been pressed, thus helping deaf people reach the door whenever the door is knocked.

\section{SYSTEM BLOCK DIAGRAM}

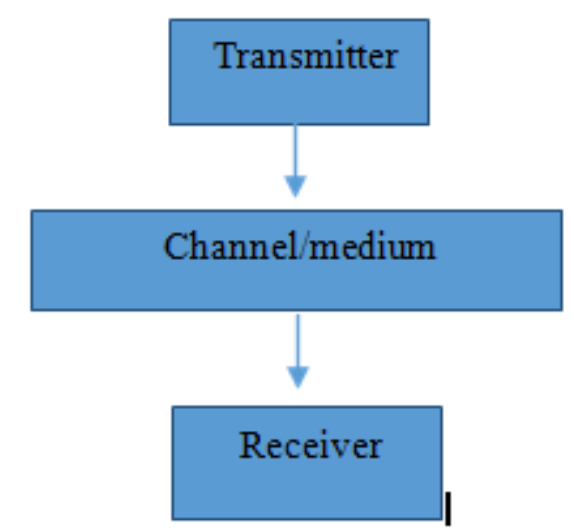

Fig.2. Block diagram of the system.

Here the transmitter contains an Arduino module connected with a doorbell along with wireless module, while the receiver's module contains Arduino, LCD display, vibrating motor/flashing LED and wireless module.

\subsection{ARDUINO NANO}

Arduino Nano has ATmega168/ATmega328P processor. Based on its processor its operating voltage is $5 \mathrm{v} / 7-9 \mathrm{v}$.It has CPU speed of $16 \mathrm{MHz}$, which is quite good. It has 8 Analog pins, 14 digital pins and 6 PWM pins. Its small size makes it quite comfortable to be used in wearable projects [5].

\subsection{Wireless Tran receiver}

NRF24L01 is a trans-receiver which works in the ISM frequency range of $2.4-2.4835 \mathrm{GHz}$. It has an on chip regulator embedded in it. When used in a power down mode 900nA of current flows through it. The main advantage of these types of trans-receiver is the power dissipation is very minimal and they are compact in size [6].

\subsection{LCD DISPLAY}

Nokia 5110 is a display unit which is used in many applications. It uses PCD8544 which is a low power CMOS LCD controller/driver. It has 48 rows and 84 columns, display data RAM of $48 \times 84$ bits. It consumes low power which is suitable for battery operated systems

\subsection{VIBRATING MOTOR/LED}

Here the vibrating motor and LED is used for the indication purpose along with LCD display. In this project the motor used was of rating, DC 3V-70mA which was light in weight and had high speed. LED of any color can be used. In this project a red colored LED was used. 


\section{FLOWCHART}

The flow of the process is shown in the figure. Here the process is divided into two segments. The region above "Medium/Channel" is transmitter's region and the region below it is receiver's region. One Arduino unit will be attached at the doorbell. The Arduino connected over there will continuously monitor whether the doorbell is pressed or not, if Yes, then send the signal "open the doorbell" via wireless module. And if the result is negative then Arduino will continuously keep reading the status of the doorbell until it is pressed. Now in the receiver's side that is the wearable device, the Arduino connected over it will check whether any signal has been detected or not, if yes then message is displayed over LCD screen along with vibrating motor and with flashing LED. And if the result is No, then keep waiting for the signal until it is send from the transmitter's side.

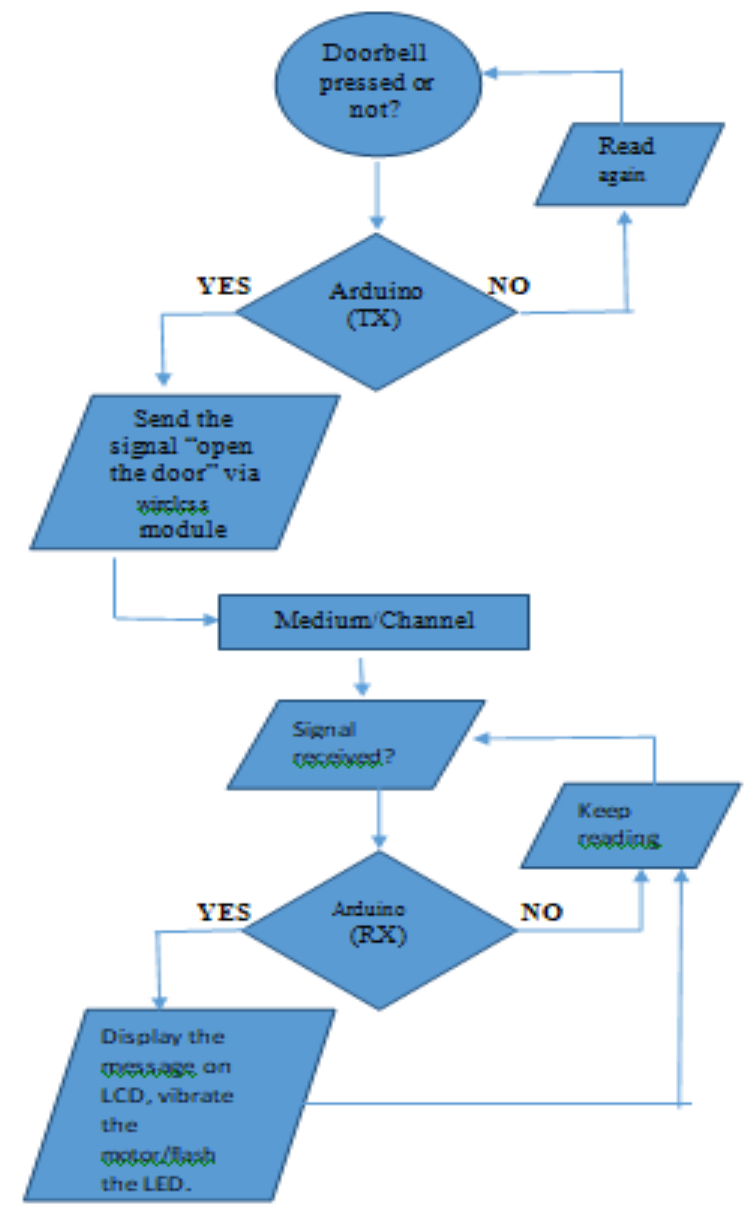

Fig.3. Proposed Doorbell notification system

\section{EXPERIMENTAL RESULTS}

Below is the schematic view of the transmitter and receiver module. Whenever the doorbell is pressed, the wireless module of transmitter module will transmit a signal to the receiver's wireless module, which as a result fetches a signal and displays a notification on the screen as "OPEN THEDOOR" with flashing LED or vibrating motor. Here in

the receiver's schematic only LED is shown as indicating device.

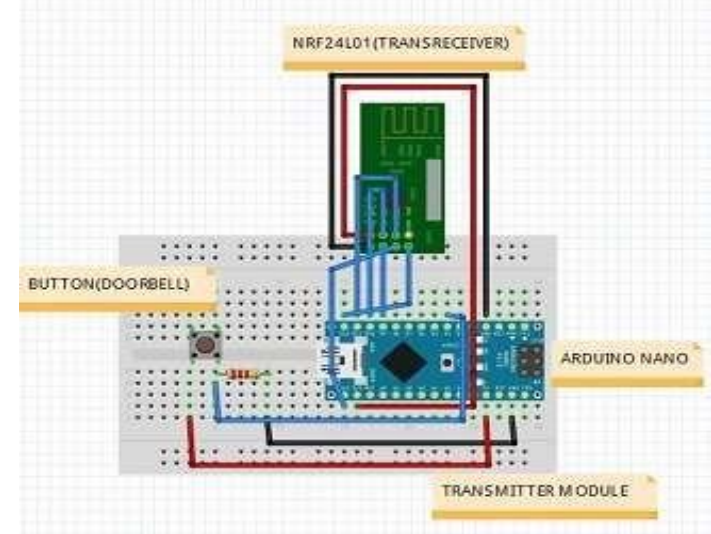

Fig. 4. Schematic of Transmitter module

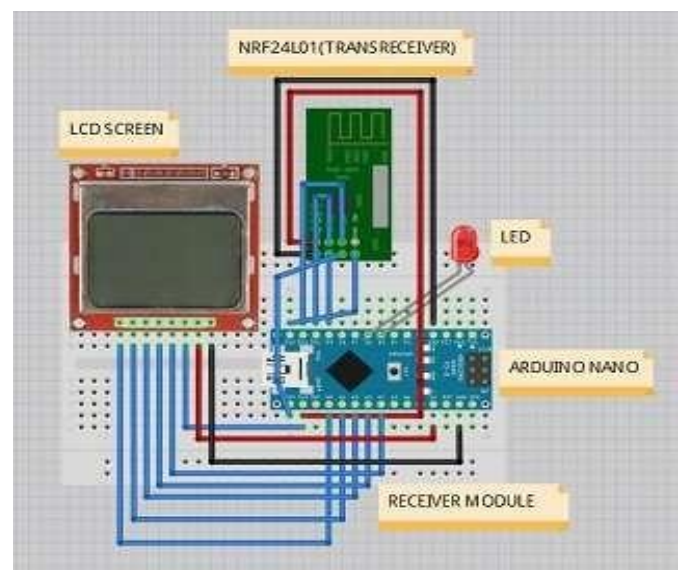

Fig .5. Schematic of Receiver module

The physical realization was a bit challenging, as the aim was to make the device as compact as possible. After few tries we came up with the best design which was suitable for our project.

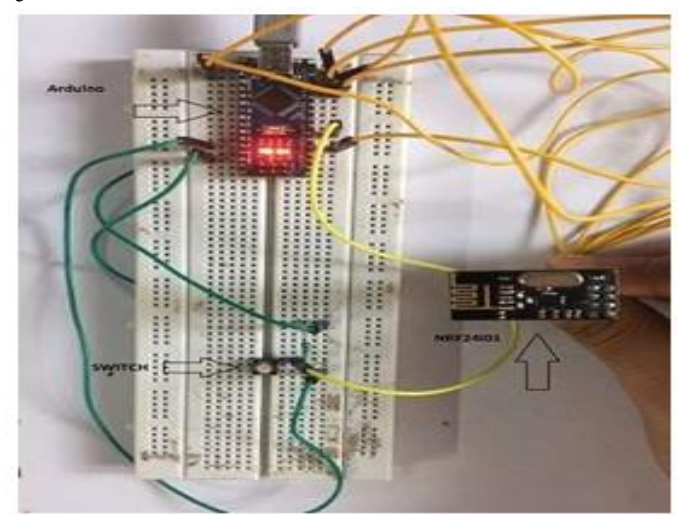

Fig 6. Transmitter module

Above is the result after final PCB implementation of receiver module. In this both vibration motor as well as LED was used for indication purpose. We see that the device is compact in size which not only makes it light in weight but also makes it very assessable and wearable. Below is the physical implementation of transmitter module. 


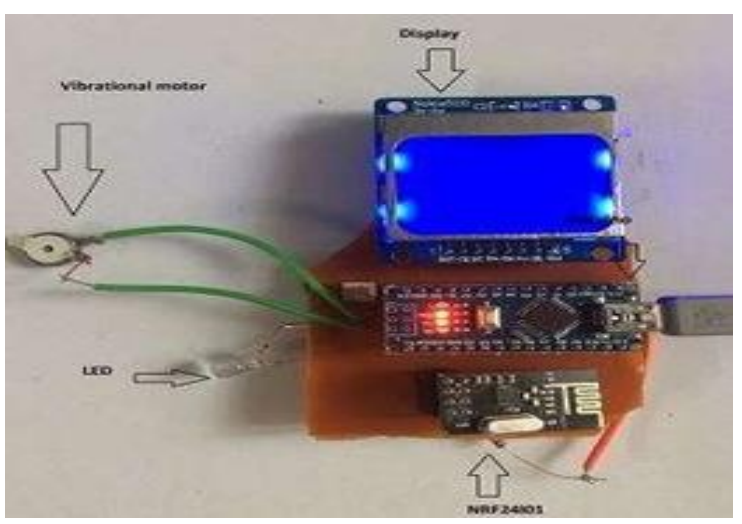

Fig 7. Receiver module

\section{CONCLUSION}

As it can be seen in the figure that when the doorbell/button was pressed immediately the message was displayed on the screen with motor vibrating and flashing led. The doorbell notification for deaf people is a practical solution has been experimentally proven to work satisfactorily. The handshaking between Arduino with various sensors has been done successfully. Thus the wearable device for deaf people was successfully designed, implemented and tested.

\section{FUTURE SCOPE}

Scope and utility of this device can be increased by adding more features in it. Adding sensor like gas sensors would allow the user to escape or call anyone of the members for the help if in case there's a fire caught up in the place or near the surrounding. Apart from adding gas sensor a noise level monitoring system can be implemented on the device, this will enable the user to respond against the noises present in his/her surroundings Not only this, it will also help the user to respond against emergency sirens like fire extinguisher truck's sound, Police van's sound or Ambulance vehicle's sound. This will keep user alert of his/her environment. Moreover an additional thing can be done to make the communication process more user friendly and feasible is that a mobile applications. Can be developed and could be installed on all the other members of the house. An additional button will be provided on the wearable module, whenever the user gets trapped in any of the situations like if the person is locked inside and is unable to open the door, then that person can press the button, which will immediately notify other members of the house about the situation of the person. This button can also be utilized for cases when user needs some kind of help from other members.

\section{REFERENCES}

1. Sriram, N., and M. Nithiyanandham. "A hand gesture recognition based communication system for silent speakers." Human Computer Interactions (ICHCI), 2013 International Conference on. IEEE, 2013.

2. Matthews, Tara, Janette Fong, and Jennifer Mankoff. "Visualizing non-speech sounds for the deaf." Proceedings of the 7th international ACM SIGACCESS conference on Computers and accessibility. ACM, 2005.

3. Jimmy Azar,Hassan Abou Saleh and Dr. M. A. Al-Alaoui. "Sound
Visualization for the Deaf," Univ. of American university of Beirut faculty of engineering and architecture, Rep. p. 3/27-6/27.

4. Munirwati Bt Anwar. "Wireless notification for hearing impaired,"M. Eng. thesis, Faculty of Electrical Engineering, Universiti Teknologi Malaysia.

5. Various Arduino boards and its comparison. [Online].Available: https://www.arduino.cc/en/Products/Compare

6. Specification about the wireless transreceiver. [Online]. Available: https://www.sparkfun.com/datasheets/Components/SMD/nR F24L01Pluss_Preliminary_Product_Specification_v1_0.pdf

7. Specification about the Nokia 5110 LCD Screen. [Online]. Available https://www.sparkfun.com/datasheets/LCD/Monochrome/No kia5110.pdf 